\title{
Adapting Load Balancing Techniques for Improving the Performance of e-Learning Educational Process
}

\author{
Ayman E. Khedr ${ }^{1}$, Amira M. Idrees ${ }^{2 *}$ \\ ${ }^{1}$ Department of Information Systems, Faculty of Computers and Information, Helwan University, Cairo, \\ Egypt. \\ ${ }^{2}$ Department of Information Systems, Faculty of Computers and Information, Fayoum University, Fayoum, \\ Egypt. \\ * Corresponding Author. Tel.: 02-01113900394; email: ami04@fayoum.edu.eg \\ Manuscript submitted November 28, 2015; accepted March 25, 2016. \\ doi: 10.17706/jcp.12.3.250-257
}

\begin{abstract}
Nowadays there is a global understanding for the importance of e-learning systems which has proved its applicability and advancement through the years especially in higher education sector. According to this increase in its importance, many educational organizations have applied e-learning process with focusing on the benefits to the student in applying this learning system. However, this increase has led to the requirements of applying a scalable, reliable, and high performance e-learning system to ensure the student's satisfaction and raising the students' education level which is the main aim of the educational process. Consequently, the role of new information technology directions is increasing for the success of the e-learning process. In this paper, we propose an adaptive educational process for e-learning systems based on applying e-learning on cloud computing environment with the support of a load balancing technique to ensure the highest available performance in the educational process and the positive impact for the student 's user satisfaction. The proposed approach has been evaluated and the results have been demonstrated in this paper.
\end{abstract}

Key words: e-Learning, load balancing techniques, system performance.

\section{Introduction}

Over the past years, e-learning has proved its positive impact on the education system for different stakeholders specially students and teachers [1]. Since e-learning has been emerged in Egypt, it has been recognized as one of the most interesting educational steps in all stages of education. However, the growth of the education system requirements has forced the need of enhancing the supportive systems such as e-learning [2]. As E-learning is currently one of the main requirements in the process of education in all its stages, therefore, a continuous improvement is always on focus. This improvement includes the main e-learning process, the system content, its continuous availability, its performance, and the stakeholders' satisfaction. A study in [3] has revealed that the e-learning is currently having a great impact on the teaching process and this impact will continuously grow in future.

As discussed in [2], since e-learning currently has a strong acceptance for both lecturers and students, then the opportunities for using e-learning should coop with this need. E-learning should become one of the continuously available systems for students, with the highest possible performance to support students in performing the required tasks and to have the highest benefits. Therefore, a focus on the system 
performance which is our focus in this research is currently an important aspect in the whole procedure.

The continuous increase in accessing e-learning resources may reveal many problem including the resources availability due to the need of continuous upgrading of these resources and extending them. One of the effective solutions that are applied for this situation is using cloud computing environments [4]. Cloud computing supports the educational organizations to provide students with the required services, moreover, it supports deploying as many tools as required to serve the students [5]-[7]. Another issue that arise according to the continuous increase in accessing e-learning is the wide number of students who may use e-learning system simultaneously which may lead to a high load on the system, thus providing a scheduling technique in e-learning is proved to an effective solution which lead to the continuous availability of the services with the maximum available performance. Consequently, providing a load balancing technique on the e-learning system that is using cloud computing environment is considered an effective solution to reveal a high impact on the success of the applied e-learning systems in the educational organizations. In this paper, we focus on applying a load balancing technique on an e-learning educational system that is available in one of the most popular universities in Egypt, Helwan University to reveal its high impact in the success of the applied system.

In this research, we have focused on enhancing the performance of e-learning in one of the most popular universities in Egypt, Helwan University, which continuously gain an increase in students' number every year. As E-learning has been applied many years ago, we aim to increase the performance of the applied system in order to be able to be balanced against the expected increase in the students number.

Load balancing ensures the improvement of the distributed system performance through the idea of restructuring the system workload with the aim of reaching the highest users' satisfaction and the maximum throughput. Load balancing techniques are applied to ensure the avoidance of the situation of having a large difference in the servers' workload [8], [9]. Several static and dynamic load balancing techniques has been discussed, some of these algorithms are Round Robin Load Balancing Algorithm, Weighted Round Robin Algorithm, Random Allocation Algorithm, and task scheduling. The following is a discussion for each algorithm [10], [11].

Round Robin Algorithm is a static load balancing technique which aims in the equal chance distribution for all servers, the service is allocated to the server according to its round in the circulated queue. Round Robin algorithm is simple in its implementation, and can provide an acceptable performance in the case of having the servers with the same capabilities to avoid less usage of the higher server's capability and over usage of lower server's capability. A weighted round robin algorithm works in the same manner as the round robin with providing a weight to each server. The weight of the server represents its priorities in being selected to provide the required services. Although this technique leads to more complexity in the computation time, however, it provides more efficient procedure as the server that has higher priority, also has higher capability, and therefore can have a prior queue role.

Random allocation load balancing technique process is to select the server randomly and is assigned for the service. Although the algorithm is simple in its implementation which was the reason to be considered in this research, however, it can provide inequality of the services distribution and the servers' load. One of the successful dynamic load balancing techniques is task scheduling, the work load is distributed based on the utilization of the servers, the servers utilization are examined referring to the total work load in the network, then the server with the least utilization is assigned to the service. This technique provides more efficient process than the random allocation technique, however, it has a considerable complexity in its computations.

The remaining of the paper is as follows, Section 2 discusses the previous work that has been performed. Section 3 discusses the proposed approach, Section 4 presents the experimental evaluation, and Section 5 
presents the conclusion and future work.

\section{Previous Works}

Different work has applied load balancing technique on the educational process, a work has been presented in [12] which applied the k-means clustering algorithm for building clusters of nodes and then apply a load balancing technique targeting the ability to handle the high requests' rate. The proposed approach in [12] has been measured in a simulator system to prove its success, however, as mentioned by the author, the system should be applied on a real system for ensuring the performance measures, moreover, the proposed approach requires to minimize the transferring in the tasks to minimize the required network cost.

Another research by [13] in which focused on a determined service in the e-learning process, this service is the files' download. The system based on building a hash table to store the information of a recently downloaded files, this table is accessed when a request is created as a primary search for the file. The target of the proposed research in [13] is to reduce the CPU time, however, the proposed approach focused only on one of the services which is the files' download while more services are proposed in the e-learning system such as uploading the assignments' solution, browsing the students' degrees, and working on a stream file. These services are not considered in the previous discussed research while they seriously affect the network traffic.

As Enhancing the educational process is one of the most effective targets in the educational system, therefore different stages have been applied for the target of reaching the highest available performance with the highest percentage of students' satisfaction. According to [14], an enhanced educational system has been proposed which aimed in adopting cloud computing in the e-learning system. The framework focused on providing different learning methods to the students which increase the student's satisfaction with ensuring the system performance. The proposed framework succeeded in providing the required satisfaction based on providing different educational services to students. As the variations of the students characteristics may require different methods of learning, the services included in the adopted framework aimed in supporting the students with different styles of learning with determining his suitable preferred methods to use it in the following learning phases. These variations of learning methods was provided by using cloud computing technology to ensure the ability of the educational system in supporting the students with a variety of successful learning methods with no limitations of the required environment capabilities. With adopting the learning systems with cloud computing, the focus was for enhancing the process.

The proposed adapted system by [14] has been applied on 1200 students in Helwan University, and has proved its success in enhancing the educational process with increasing the students' satisfaction. However, the previous experiment did not consider the high network traffic situation. When applying the enhanced educational system in 20 minutes exam for the 1200 students, this has led to a high traffic in the system during a specified period of time. It has been noticed that this situation has seriously reduced the system performance. This paper focuses on the e-learning process based on the adoption of cloud computing with the target of enhancing the system performance and availability. The proposed approach in this paper aims to apply a load balancing technique on the adopted e-learning system to overcome the performance problem during the high services rate hours. Section 3 presents the proposed enhancement.

\section{Proposed Methodology}

As discussed in Section 2, adopting cloud computing technology on the e-courses increased the satisfaction in the e-learning process but confronted the problem of performance during peak time. As an 
adaptive stage for solving this situation, a load balancing technique has been proposed for the enhanced educational process. Load balancing is a vital research field in the cloud computing environment which can provide a solution for many problems considering the performance, availability, and reliability. In peak time, users require to have the same services' performance with not considering the high services rate in the whole network, therefore, providing a balancing technique to balance the workload for the users lead to achieving the maximum throughput with attaining the minimum response time.

In this research, we have applied two load balancing techniques, they are random allocation, and task scheduling techniques, each has its own privileges and limitations. Sections 3.1, and 3.2 discusses the two approaches in details.

\subsection{Enhancing Educational Process Based on Random Allocation Load Balancing}

In this phase, the static load balancing "Random Allocation Load balancing" technique has been applied for the proposed enhancement in the educational process of the e-learning system [10], [15]. One of the benefits which was the motivation for applying this algorithm is that it is easy in its implementation, it also does not need for special configuration requirements. Fig. 1 presents a flow chart for by introducing the load balancing approach based on random allocation algorithm.

To explore the enhancement on the system performance, the random allocation load balancing technique has been developed on the enhanced educational system in 20 minutes exam for the 1200 students which has led to a high traffic in the system during a specified period of time. The system has proved its advancement on the previous system situation which is explored in the calculated measures. However, Targeting to generalize the educational process by applying the enhanced system on different courses with maintaining higher performance in different situations, when applying the system on 6000 students in a limited determined time period for four different courses, the system had a very serious situation in decreasing performance according to the strong need of service availability to all users (students). For more stability and for the target of finding the best system performance and throughput, we have applied one of the most successful dynamic load balancing technique which is task scheduling as will be described in the next Section 3.2.

\subsection{Enhancing Educational Process Based on Random Allocation Load Balancing}

Based on the drawbacks that raised in applying the static random allocation load balancing technique, and with targeting more orientation to the services and an increase to the system performance, task scheduling technique is applied for adapting the enhanced educational process which in turn leads to students' satisfaction [16], [17].

One of the benefits in applying a dynamic load balancing technique that its nature is to consider the attribute of each node and the available bandwidth of the network. The task is scheduled according to the information that is determined for each client. This situation requires continuous monitoring to the network traffic, assigned schedule, and the nodes' activities. Although this approach is harder in its implementation, however, when applying the system on 6000 students in a limited for the four courses with determined time period according to our applied approach, the has proved to provide higher performance and higher throughput as will be shown in the measurements in Section 4.

\section{Experimental Evaluation for Applying Both Presented Load Balancing Techniques}

The target of this experiment is to generalize the proposed approach on the e-learning educational process over a large number of e-courses with high rate of students in all stages of the educational process with the highest applicable system performance and students' satisfaction. Therefore, the previously discussed load balancing techniques have been developed and applied on 6000 students for four courses in 
20 minutes exam in order to measure the servers' utilization in high services' rate which explore the system performance. The results has showed the advance of the task schedule technique. However, besides the higher complexity in the implementation of task scheduling load balancing algorithm, another drawback is the high power consumption which is not our focus in this research. The results are shown in the comparative measurements in Fig. 1 to Fig. 7.

Different measures can be applied to explore the performance of the system [18], we have selected some of these measures. Fig. 1 measure the response Time which determines the time required for the server to respond to the request starting when it received this request. According to Fig. 1, it is shown that task scheduling has the lead as it has less response time. Another measure is the latency which measure the time required to assign the required service to a server in the network, according to Fig. 2, random allocation has more stable latency than task scheduling according to the complexity in computation that is required in task scheduling.

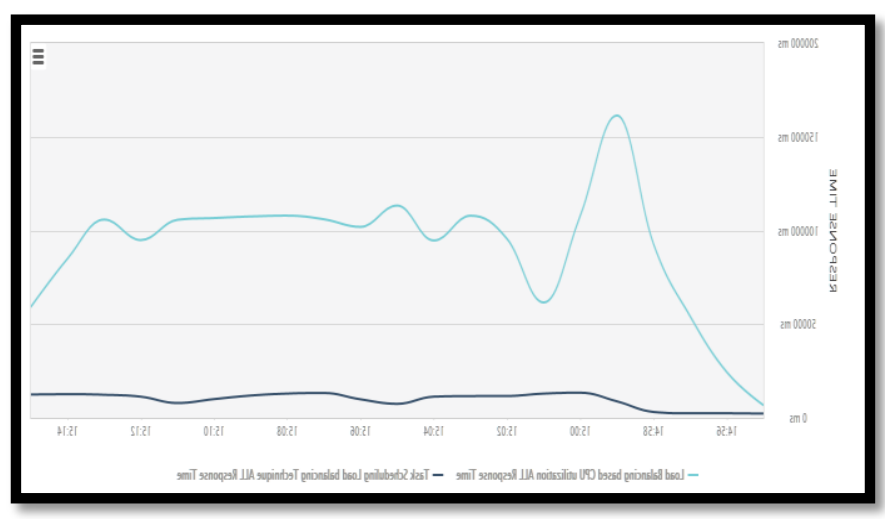

Fig. 1. Response time comparison between random allocation load balancing and task scheduling techniques in the educational process based on cloud computing.

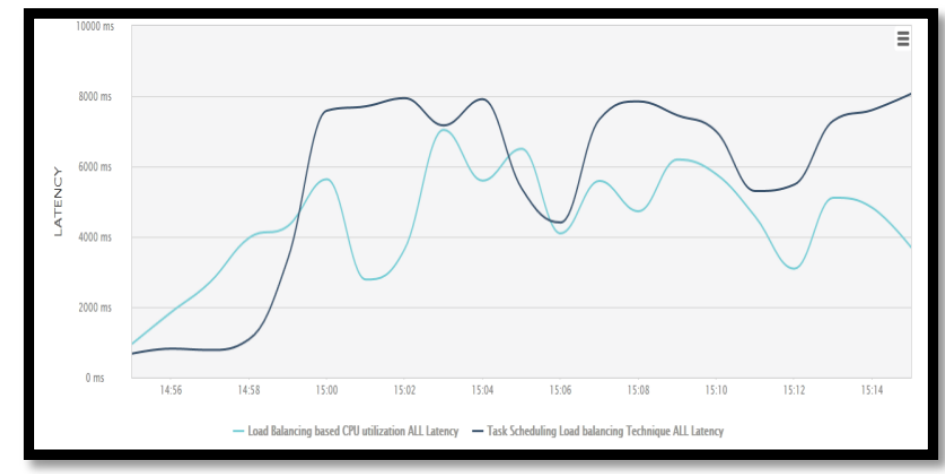

Fig. 2. Latency comparison between random allocation load balancing and task scheduling techniques in the educational process based on cloud computing.

Network Bandwidth illustrated in Fig. 3 measures the usage of bandwidth is derived from several parameters including the required computation, the traffic rate, and the nature of services. It is determined that task scheduling has higher bandwidth usage as it utilizes the network resources for providing the required services. Another measure is the Hit Rate illustrated in Fig. 4 which determines the number of completed required services, it is related to the user satisfaction which increases by the higher hit rate provided by the task scheduling. And finally the Errors Rate is presented in Fig. 5 measures the load deviation in the network, the lower the error rate provides higher load balancing in the network, as shown in Fig. 5, task scheduling has almost zero error rate. 


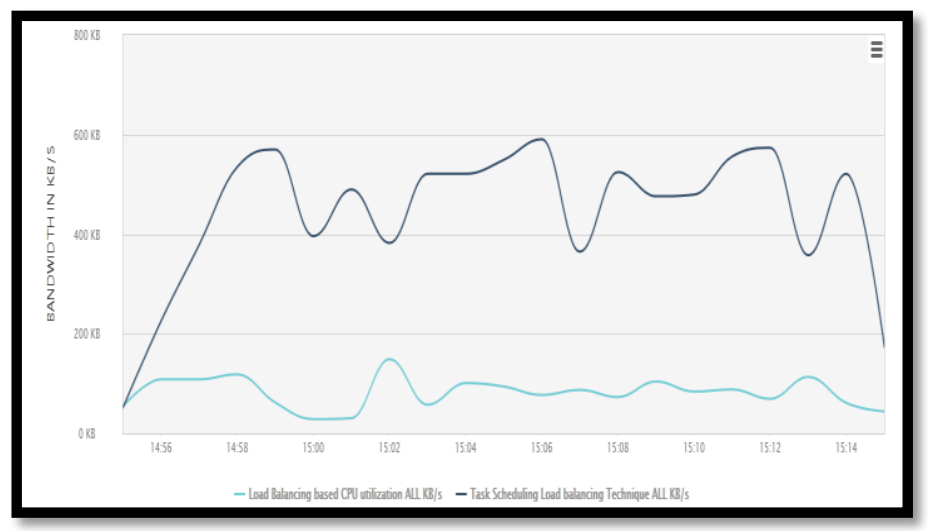

Fig. 3. Bandwidth comparison between random allocation load balancing and task scheduling techniques in the educational process based on cloud computing.

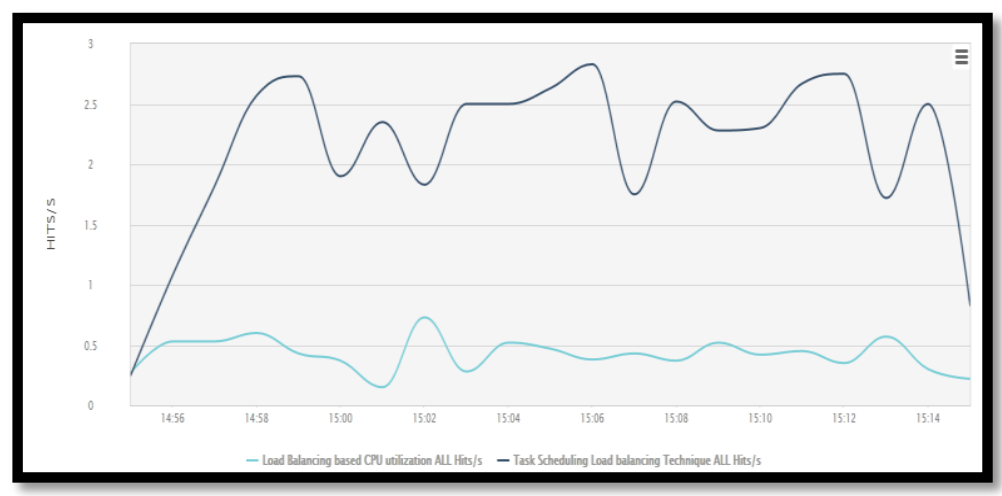

Fig. 4. Hits rate comparison between random allocation load balancing and task scheduling techniques in the educational process based on cloud computing.

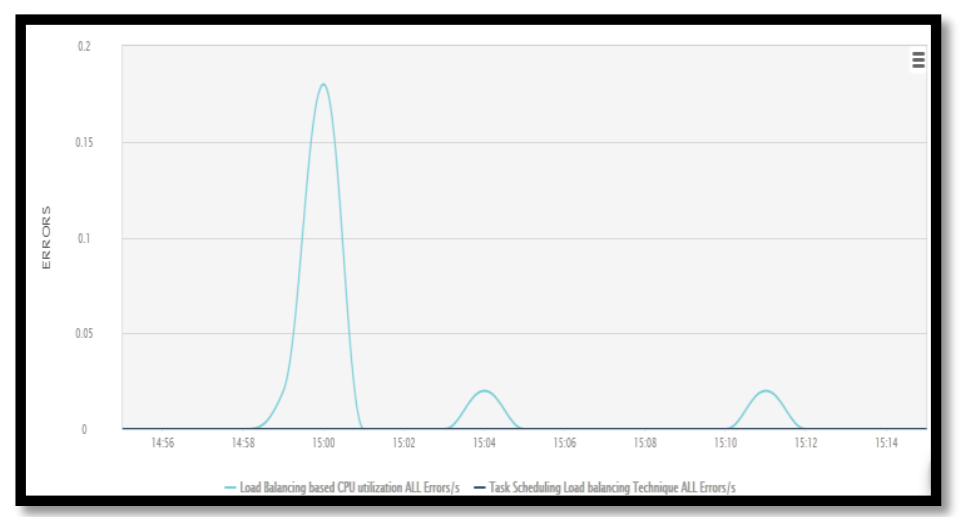

Fig. 5. Errors rate comparison between random allocation load balancing and task scheduling techniques in the educational process based on cloud computing.

\section{Conclusion and Future Work}

In this paper, we presented an enhancement of the educational process over the cloud computing environment based on applying a load balancing technique for the target of increasing the system performance in the high services' rate duration time, which consequently lead to an increase the students' satisfaction. The proposed approach has been evaluated by applying two load balancing techniques, they are random allocation and task scheduling. Both situations have been evaluated and the results have been presented which explore the pros and cons for each algorithm and its impact on the whole process. 
Although applying the presented experiments has explored the positive impact on the proposed approach, however, more enhancements can be proposed. One of these enhancement is to consider the power consumption rate during the process, another direction is to apply the proposed system in more crowded system to ensure its reliability. Moreover, exploring more load balancing techniques should be performed for the target to find the suitable algorithm for different situations with providing an integration technique that consider the selection of the best algorithm according to the current network situation.

\section{Acknowledgment}

With deep appreciation, the authors would like to thank Eng. Hesham ElMasry for his support and cooperation to this publication.

\section{References}

[1] Nichols, M. E-Learning in Context. Retrieved 2008, from: http://akoaotearoa.ac.nz/sites/default/files/ng/group-661/n877-1---e-learning-in-context.pdf

[2] Rashad, A. S. (June 2013). The impact of e-learning system on conventional teaching environment. Proceedings of International Conference on the Future of Education, Florence, Italy.

[3] Golden, S., McCrone, T., Walker, M., \& Rudd, P. (2006). Impact of e-learning in further education: Survey of scale and breadth. National Foundation for Educational Research.

[4] Popovic, K., \& Hocenski, Z. (2010). Cloud Computing Security Issues and Challenge, 344-349.

[5] Pocatilu, P. (2010). Cloud computing benefits for e-learning solutions. Oeconomics of Knowledge, 2(1), 9-14.

[6] Qian, L., Luo, Z., Du, Y., \& Guo, L., (2009). Cloud computing: An overview. Springer-Verlag.

[7] Khedr, A. E., Kholeif, S. A., \& Hessen, S. H. (March 2015). Adoption of cloud computing framework in higher education to enhance educational process. International Journal of Innovative Research in Computer Science and Technology, 3(3), 150 -156.

[8] Zhao, Y., \& Huang, W. (2009). Adaptive distributed load balancing algorithm based on live migration of virtual machines in cloud. Proceedings of 5th International Joint Conference on INC, IMS and IDC.

[9] Zhang, Z., \& Zhang, X. (2010). A Comparative study into distributed load balancing algorithms for cloud computing. Proceedings of 2nd International Conference on Industrial Mechatronics and Automation.

[10] Vakkalanka, S. (Sept-Oct. 2012). Classification of job scheduling algorithms for balancing load on web servers. International Journal of Modern Engineering Research, 2(5), 3679-3683.

[11] Katyal, M., \& Mishra, A. (December 2013). A comparative study of load balancing algorithms in cloud computing environment. International Journal of Distributed and Cloud Computing, 1(2).

[12] Rajarajeswari, S. (2013). ECLB: A novel exhaustive criterion based load balancing algorithm for e-learning platform by data grid technologies. Int. J. Advanced Networking and Applications, 4(6), 1786-1792.

[13] Dominic, M., \& Francis, S. (June-July 2014). Load balancing using peers in an e-learning environment. International Journal of Computer Science and Business Informatics, 14(1).

[14] Khedr, A. E., Kholeif, S. A., \& Hessen, S. H. (March 2015). Enhanced cloud computing framework to improve the educational process in higher education: A case study of Helwan University in Egypt, International Journal of Computers \& Technology, 14(6).

[15] Das, S. K., Harvey, D. J., \& Biswas, R. (June 2002). Adaptive load balancing algorithms using symmetric broadcast networks. Journal of Parallel and Distributed Computing, 62(6), 1042-1068.

[16] Fang, Y., Wang, F., \& Ge, J. (2010). Lecture Notes in Computer Science, 6318, 271-277.

[17] Li, K., Xu, G., Zhao, G., Dong, Y. \& Wang, D. (2011). Cloud task scheduling based on load balancing ant 
colony optimization. Proceedings of 2011 Sixth Annual China Grid Conference.

[18] Keerthika, P., \& Kasthuri, N. (2013). A hybrid scheduling algorithm with load balancing for computational grid. International Journal of Advanced Science and Technology, 58, 13-28.

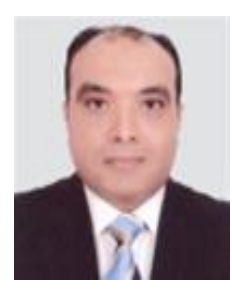

Ayman E. Khedr is an associate professor, and he is also the head of Information Systems Department, Faculty of Computers and Information Technology, Future University in Egypt. He is the supervisor of the Quality Assurance Department and a member of continuing education board. He has worked at the Faculty of Computers and Information, Helwan University in Egypt, and the general manager of Helwan e-Learning Center. His research is concentrated around the themes (scientific) data and model management, data mining, bioinformatics and cloud computing.

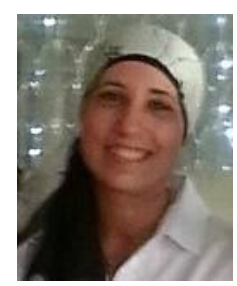

Amira M. Idrees is an associate professor in the Faculty of Computers and Information, Fayoum University. Her research interests include knowledge discovery, text mining, opinion mining, sentimental analysis, cloud computing, software engineering, and data warehousing. 